\title{
IATROGENIC DISLOCATION OF THE LOWER WISDOM TOOTH TO THE PERIMANDIBULAR SPACE - A CASE REPORT
}

\author{
Miłosz Ambicki, Bogumił Lewandowski \\ Department of Maxillo-Facial Surgery, F. Chopin Clinical Provincial Hospital in Rzeszow, Poland
}

\begin{abstract}
Iatrogenic dislocation of the lower wisdom tooth to the bottom of the oral cavity and neck is a rare complication that may occur during surgical extraction, usually causing inflammation and abscesses, and therefore it requires specialist treatment in a hospital in the Department of Maxillo-Facial Surgery. The case described in this paper required surgical removal of the displaced tooth and evacuation of the abscess from the extraoral approach. The patient had uneventful perioperative course, no postoperative complications were observed, and the healing proceeded in a normal way. During three outpatient follow-ups carried out at intervals of one month, no abnormalities in healing were found.
\end{abstract}

KEY WORDS: wisdom teeth, surgical extractions of third molars, accidental iatrogenic complication, treatment of complications, displaced tooth.

J Stoma 2019; 72, 3: 142-146

DOI: https://doi.org/10.5114/jos.2019.87535

\section{INTRODUCTION}

Extractions of retained wisdom teeth are common procedures in dental practice as well as in dental and maxillo-facial surgery worldwide. Due to the muchdelayed development of the lower third molars in relation to the remaining teeth, they are the most common retained teeth which is often an indication for their surgical extraction. These treatments are usually elective after proper examination of the patient, which consists of assessment of the teeth positions on the base of pantomographic X-ray and planning of the operating method and technique. Postoperative care is also important $[1,8,15]$.

During and after the surgical extraction of the wisdom tooth, different complications may occur, both general and local. The list of possible complications is very long. Among these complications, iatrogenic displacement of the tooth or its fragment to the perimaxillary tissues and anatomical structures is definitely worth mentioning. This includes: dislocation of the tooth to the maxillary sinus, to the mandibular canal, and to the tissues of the bottom of the oral cavity and the cheek $[3,4]$. One of the most frequently occurring complications in this group is the displacement of the upper third molar or its fragment into the maxillary sinus [14]. Accidental insertion of the lower wisdom tooth or its fragment into the tissues of the mouth and neck, i.e. sublingual, submandibular, or pterygomandibular space, is rarely presented in the literature, and then usually as case studies $[2,5,12,13]$. The cortical bone plate that covers the roots of the lower molars from the lingual side is very thin. Its partial lack in the area of the third molars may be favourable to different complications and dislocations of the tooth to the surrounding soft tissues [6, 7, 9-11].

\section{JOURNAL OF} STOMATOLOGY CZASOPISMO STOMATOLOGICZNE

AdDresS FOR CORRESPONDENCE: Miłosz Ambicki, Department of Maxillo-Facial Surgery, F. Chopin Clinical Provincial Hospital in Rzeszow, Poland, e-mail: milosz.ambicki@gmail.com

ReCEIVED: 12.04.2019 • ACCEPTED: 05.08.2019 • Published: 08.08.2019 
This paper presents a rarely described complication that occurred in a patient during the extraction of the lower left wisdom tooth (tooth 38) and consisted of its accidental insertion to surrounding perimandibular tissues of the bottom of the oral cavity and the submandibular space.

\section{CASE PRESENTATION}

The patient, aged 23 years, reported to the Clinic of Maxillo-Facial Surgery, F. Chopin Clinical Provincial Hospital in Rzeszow to treat complications after unsuccessful extraction of a lower wisdom tooth caused by insertion of tooth 38 into the tissues of the bottom of the oral cavity. Medical history revealed that the indications for extraction were occasional symptoms and inflammation in the area of the partially erupted lower wisdom tooth on the left. Ailments subsided after conservative local treatment and antibiotic therapy. Tooth 38 was qualified for extraction after analysing the pantomographic X-ray with the consent of the patient (Figure 1). During the removal of tooth 38 technical difficulties occurred. As a result of the surgery the tooth was dislocated to the perimandibular tissues. The procedure and further attempts to remove the tooth were abandoned. Immediately after the surgery the physician performing the procedure administered an antibiotic (clindamycin) in a dose of $3 \times 300 \mathrm{mg}$ and took a panoramic radiograph (Figure 2 ). On the day after the procedure gradually increasing pain, lock-jaw, swelling of the submandibular region on the left, and then difficulty in swallowing occurred. An additional cone beam computed tomography (CBCT) (Figures 3 and 4) examination was performed for more accurate diagnosis, and the patient was urgently referred to the Clinic of Maxillofacial Surgery, F. Chopin Clinical Provincial Hospital in Rzeszow. On admission to the clinic, the patient was found to have hard, tender, board-like, and non-movable swelling in the left submandibular area. She also reported increasing difficulties in swallowing both solid and liquid food and difficulty in opening the jaws at about $1 \mathrm{~cm}$. A continuation of antibiotic therapy was ordered, along with analgesics and anti-oedema medication. On the basis of a clinical examination and $\mathrm{X}$-ray imaging, the patient was qualified and prepared for the surgical extraction of the displaced tooth 38 under general anaesthesia. The abscess with the presence of the tooth was localised from the extra-oral approach, in the left submandibular region. The abscess was drained and then the tooth was extra-oral, which took place on the inside, on the lingual side of the mandible (Figures 5 and 6). After the operation passive drainage was used for 24 hours. On the third day after the procedure, the patient was discharged home in good local and general condition with a recommendation to continue antibiotic therapy.
On the seventh day after the procedure, the patient reported for follow-up. A slight swelling of the operated area and gradually disappearing lock-jaw without pain continued. The patient did not become feverish. Clinical examination showed normal healing without symptoms of inflammatory infiltration in the oral cavity and perimandibular tissues. Antibiotic therapy was continued.

A follow-up in 10 days was recommended, at which the patient did not report any disturbing symptoms. Swelling and lock-jaw subsided. The wound healed well.

\section{DISCUSSION}

Only a few publications in the literature concern accidental insertion of the wisdom tooth or its parts into the perimandibular tissues as a complication during removal of wisdom teeth $[2,5,6,10,11,12-14]$. According to Brauer's report this complication is estimated at about 1\%; however, in the literature there is no detailed research on this subject [3]. The most common cause is iatrogenic fracture or damage of the cortical layer of the alveolar bone in the region of the extracted wisdom tooth due to use of excessive force or improper surgical technique $[2,11,13]$. Unskilful use of the straight tooth elevator or luxator may cause uncontrolled, accidental displacement of the tooth beyond the alveolus $[4,8,15]$. Significant risk factors for complications

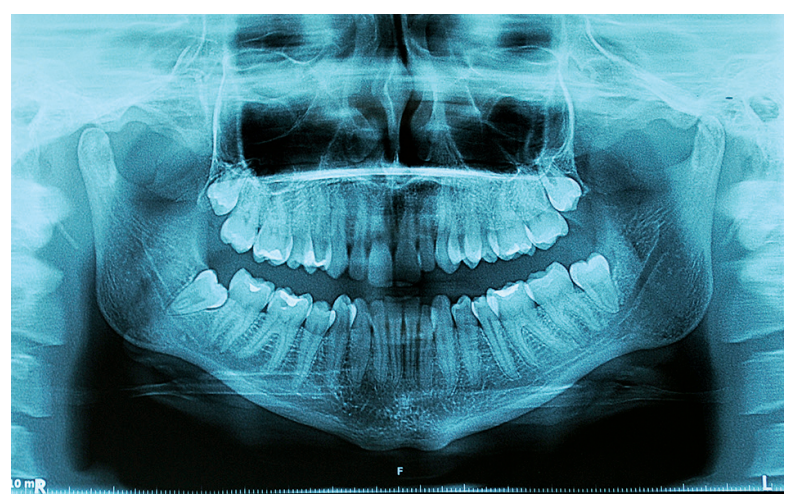

FIGURE 1. Before extraction of tooth 38

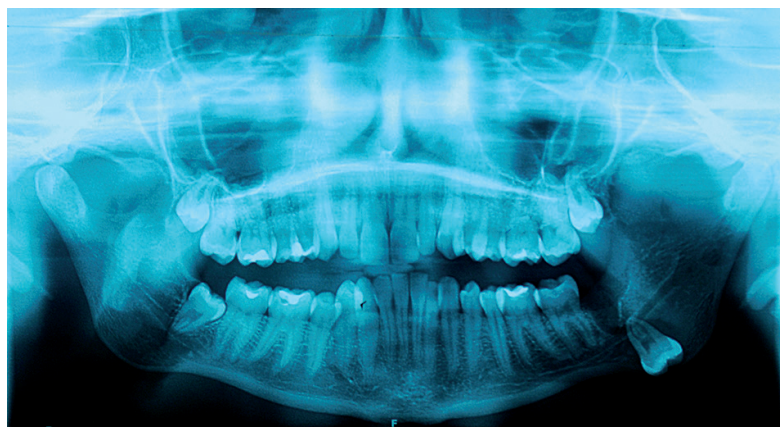

FIGURE 2. After unsuccessful extraction of tooth 38 . Displaced tooth 38 can be seen 


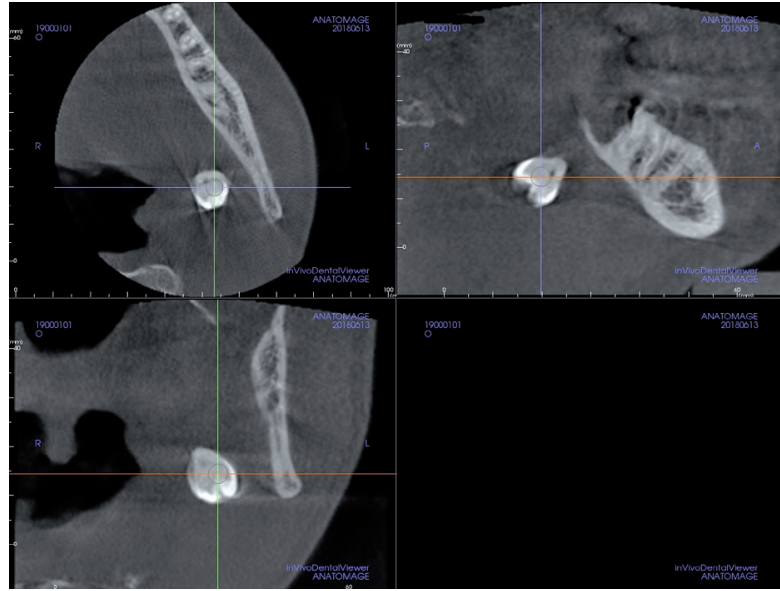

FIGURE 3. Cone beam computed tomography scan. Displaced tooth 38 can be seen

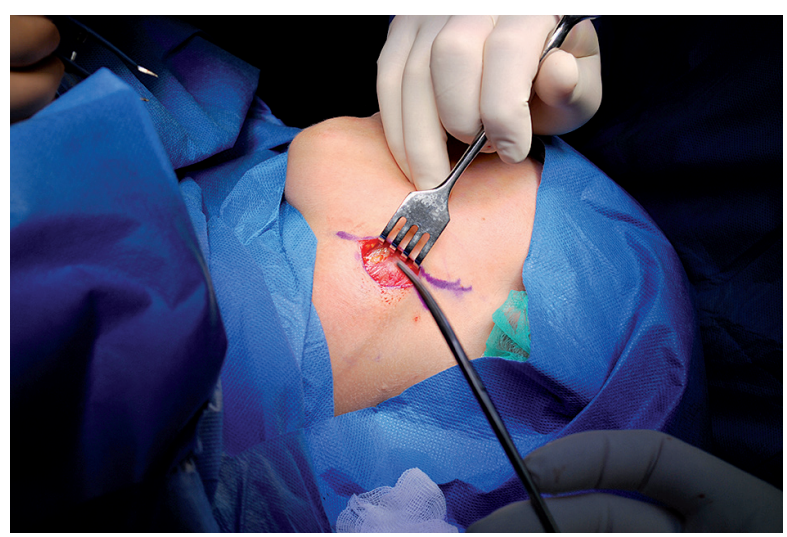

FIGURE 5. Extra-oral approach in left submandibular region

are: the anatomical structure of the mandible in this area and defects of the cortical layer from the lingual side of the alveolar bone in the mandible, distal and lingual location of the lower wisdom tooth, concavity and blocking of roots of the retained tooth, and the patient's age. The reason for the presence of "cavities" in the cortical lingual layer of the mandible may be idiopathic or iatrogenic, and can be associated with inflammatory processes or the presence of cysts in this area. Idiopathic disruption of bone continuity is usually small. In this site bone offers weaker resistance when removing the tooth, predisposing to its displacement in this direction [15].

Surgical removal of the lower wisdom tooth involves using lesser or greater strength, sharp surgical instruments, and rotary tools, which is why one cannot omit the iatrogenic factor associated with the improper use of surgical instruments. Often lack of experience and intuition, inappropriate technique, excessive strength, as well as the wrong point of its application can lead to fracture of the alveolus or fracture of the mandibular body, which facilitates the displacement of the extracted

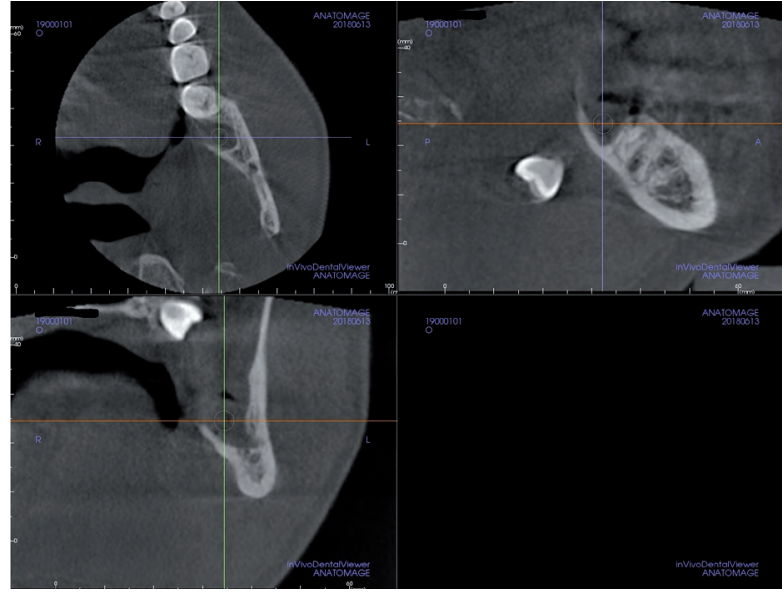

FIGURE 4. Cone beam computed tomography scan. Alveolus 38

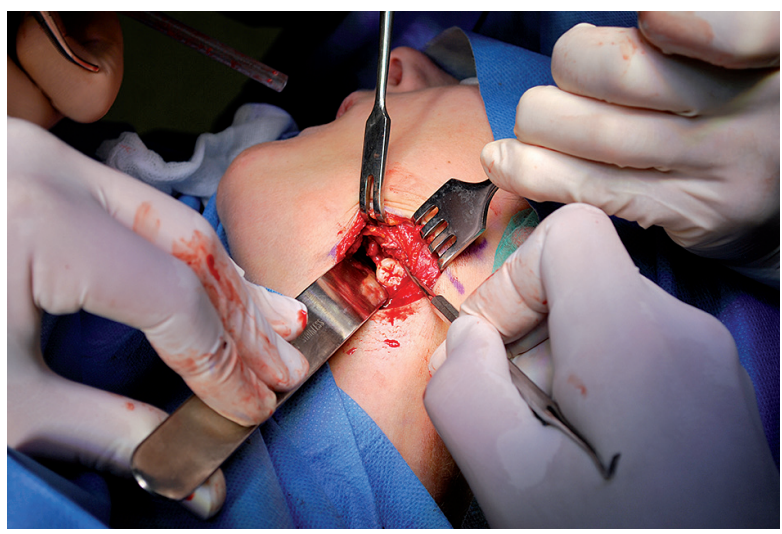

FIGURE 6. Extra-oral extraction of dislocated tooth 38 in left submandibular region

tooth $[2,13]$. In some cases, there is a need for osteotomy on the lingual side to expose the tooth crown. The medial or lingual position of a retained wisdom tooth requires cutting off the tooth crown from the root. The use of rotational tools carries the risk of damaging them and breaking or piercing the bone. Wisdom teeth in the distal lingual position with abnormalities in the anatomical structure of the root and the previously mentioned discrepancies in the anatomical structure of the surgical site constitute an additional risk of postoperative complications $[4,8,15]$.

Before the surgery is planned a physical examination and interview should be carefully performed. It should also be extended with the necessary radiological diagnostics to assess possible difficulties resulting from the position of the retained tooth and its relation to the lower alveolar nerve canal and adjacent teeth in the aspect of possible complications. In some cases, in addition to the pantomographic X-ray, it is advisable to perform a CBCT examination, which additionally presents the projection of the retained tooth in three dimensions [7]. It allows the assessment of the position 
of the tooth in relation to important anatomical structures more accurately than via panoramic radiograph.

When starting the surgical procedure of removal of the wisdom tooth the patient should be informed about the method and technique of the procedure, potential consequences, and postoperative complications that may occur. In the case of complications the patient should be informed about them immediately after they took place.

The literature describes a number of surgical methods allowing the procedure to be performed efficiently, to rule out potential postoperative complications. In some cases, it is advisable to cut and separate the crown of the tooth. Some authors recommend putting pressure on the lingual margin of the alveolar region of the mandible while elevating the tooth. This prevents its displacement towards the tongue and the bottom of the mouth and reduces the likelihood of breaking the lingual lamina $[1,8]$. Another method is to place a raspatory or a retractor between the detached mucoperiosteal flap and the bone of the alveolar part of the mandible on the lingual side optimally convex of the tool to the tooth being removed [8]. This procedure prevents the tooth from moving towards the bottom of the oral cavity, and enables the tooth an extraction tool to be used outside the alveolus. In a situation in which a complication occurs in the form of a tooth or a part of it being inserted into the tissues of the bottom of the oral cavity, the authors recommend that dentists with no surgical experience refrain from attempting to remove the already dislocated tooth.

These attempts are often difficult and lead to further displacement of the tooth into the tissues. During the preparation period the treatment as well as possible complications and consequences of the procedure should be presented to the patient. The patient should be informed about the occurrence of complications and then referred to the reference centre for specialist treatment. A referral to a reference centre should contain relevant information about the tooth being extracted, e.g. about the approximate size of its fragment, the circumstances of the complications, and radiological documentation [1, 15]. Referral should be in urgent mode. If the delay cannot be avoided, e.g. due to the considerable distance between medical offices, the dentist should dress the wound with a surgical dressing and introduce antibiotic therapy.

The data from the literature is not consistent as to the time and urgency of extracting the tooth displaced into the surrounding tissues. Some authors suggest postponement of surgery for up to several weeks. Postponement of the procedure is aimed at achieving fibrosis of tissues surrounding the displaced tooth or its fragment, which can stabilise its position and in the future facilitate extraction during surgery [13]. In that case strict control should be continued because the inserted material is treated as a foreign body that can move deep- er into the anatomical structures and impede its subsequent extraction [6]. Postponing surgery can often lead to the formation of an abscess and cause not only local complications like pain, swelling, lock-jaw, or dysphagia but also general inflammatory reaction $[3,7,9]$. Kose et al. [6] suggest removal of the dislocated tooth as soon as it is possible.

Aznar-Arasa et al. believe that the treatment of a tooth or a part of it that has been displaced depends on its size and location [2]. If the fragment is smaller than $5 \mathrm{~mm}$, patients usually do not report any complaints, and there are no clinical symptoms. The authors described a case of a patient with a fragment of a root dislocated into the perimaxillary tissues. During the five-year follow-up no clinical symptoms in the form of inflammation or other similar reaction occurred. In these situations, no surgical treatment is required. The displacement of larger fragments $(>5 \mathrm{~mm}$ ) or the whole tooth usually result in fast proceeding inflammatory complications and the need for surgical treatment. In the author's opinion treatment under general anaesthesia or local anaesthesia with intravenous sedation should be performed. This avoids the patient's involuntary movements that make the procedure difficult and increase the risk of further complications $[8,15]$.

The surgical approach in each case should be planned individually, depending on the location of the tooth or its fragment $[1,3,4,8,15]$. The most common intraoral approach in the case of dislocation to the tissues of the oral cavity and the surrounding sublingual area is the incision and mobilisation of the envelope lingual muco-periosteal flap from the mandibular ramus to the premolar area $[1,15]$. However, this technique gives limited access and insight into the tissues of the mouth due to the mylohyoid muscle, which is located in this area [2, 15]. In the case of dislocating the tooth into the deeper locations, e.g. to the submandibular region, extraoral or intraoral approach or both are required [15]. In the described case the decision about the extra-oral surgical approach was made on the basis of clinical examination and assessment of the panoramic X-ray and CBCT in terms of the location of a displaced tooth in the surrounding tissues. The patient underwent the procedure well, and no postoperative complications were observed. Healing was normal. Three outpatient follow-ups were performed at monthly intervals, which did not reveal any abnormalities.

\section{CONCLUSIONS}

Complications associated with the surgical extraction of retained wisdom teeth occur frequently in dental practice, but in many cases they can be prevented by proper preparation of the patient for the procedure and evaluation of the difficulties resulting from the clinical-anatomical location of the retained tooth. The rich 
radiological symptomatology of retained lower wisdom teeth additionally confirms the need for individual planning of surgeries with regard to possible difficulties, as well as assessing them according to one's competence and training $[8,13]$.

\section{CONFLICT OF INTEREST}

The authors declare no potential conflicts of interest with respect to the research, authorship, and/or publication of this article.

\section{References}

1. Asanami S, Kosazaki Y. Expert third molar extractions. Tokyo, Chicago: Quintessece Pub. Co.; 1990.

2. Aznar-Arasa L, Figueiredo R, Gay-Escoda C. Iatrogenic displacement of lower third molar roots into the sublingual space: report of 6 cases. J Oral Maxillofac Surg 2012; 70: e107-e115.

3. Brauer HU. Unusual complications associated with third molar surgery: a systematic review. Quintessence Int 2009; 40: 565-570.

4. Czechowska E, Rydzewska-Lipńska M, Szubert P, Sokalski J. Complications during the surgical wisdom tooth removal - case report. Dental Forum 2013; 41: 119-122.

5. I-Yueh H, Chao-Ming C, Sung-Wen C, Chia-Fu Y, Chung-Ho C, Chun-Min C. Surgical management of accidentally displaced mandibular third molar into the pterygomandibular space: a case report. Kaohsiung J Med Sci 2007; 23: 370-374.

6. Kose I, Koparal M, Gunes N, et al. Displaced lower third molar tooth into the submandibular space: two case reports. J Nat Sc Biol Med 2014; 5: 482-484.

7. Lee D, Ishii S, Yakushiji N. Displacement of maxillary third molar into the lateral pharyngeal space. J Oral Maxillofac Surg 2013; 71: 1653-1657.

8. Peterson LJ, Ellis E, Hupp JR, Tucker MR. Oral and maxillofacial surgery. Polish Edition: Pogorzelska-Stronczak Z. Lublin: Czelej; 2001.

9. Pippi R, Perfetti G. Lingual displacement of an entire lower third molar. Report of a case with suggestions for prevention and management. Minerva Stomatol 2002; 51: 263-266.

10. Satnam SJ, Vidya R, Sachin KR. Intraoral management of displaced root into submandibular space under local anaesthesia - a case report and review of literature. Saud Dent J 2014; 26: 181-184.

11. Silveira RJ, Garcia RR, Botelho TL, Franco A, Silva RF. Accidental displacement of third molar into the sublingual space: a case report. J Oral Maxillofac Res 2014; 5: e5.

12. Suer BT, Kocyigit ID, Ortakoglu K. Iatrogenic displacement of impacted mandibular third molar into the pterygomandibular space: a case report. Oral Health Dent Manag 2014; 13: 179-182.

13. Sufeng Z, Zheng H, Tengyu G, Lanzhu H. Intraoral management of iatrogenically displaced lower third molar roots in the sublingual space: a report of 2 cases. Int J Clin Exp Med 2015; 8: 1959119595.

14. Sverzut CE, Trivellato AE, Lopes LMF, Ferraz EP, Sverzut AT. Accidental displacement of impacted maxillary third molar: a case report. Braz Dent J 2005; 16: 167-170.

15. Tetsch P, Wagner W. Operacyjne usuwanie zęba mądrości. Warszawa: Wydawnictwo Medyczne Sanmedica; 1994. 